\title{
Early renal biopsy in acute renal failure - Expect the unexpected
}

\author{
Prashant G Mankar ${ }^{1, *}$, Sunil Deshpande ${ }^{2}$ \\ ${ }^{\mathbf{1}}$ Associate Professor, Dept. of Pathology, Dr. Panjabrao Deshmukh Memorial Medical College, Amravati, Maharashtra, \\ ${ }^{2}$ Consultant Nephropathologist, KEM Hospital, Pune, Maharashtra, India
}

*Corresponding Author:

Email: drprashantmankar@gmail.com

Received: $18^{\text {th }}$ January, 2018

Accepted: $22^{\text {nd }}$ February, 2018

\begin{abstract}
Present study was carried out to determine the contribution of early renal biopsy in diagnosis and management of acute renal failure (ARF) cases suspected on clinical grounds to be other than acute tubular necrosis (non ATN-ARF). Total 37 cases fulfilled the inclusion criteria. In 20 out of 37 (54.05\%) cases the clinical diagnosis was in concordance with biopsy diagnosis. In 13 out of $37(35.13 \%)$ cases the clinical diagnosis was in discordance with biopsy diagnosis which altered the management of the patient. Remaining 4 out of $37(10.81 \%)$ cases were not diagnosed clinically and diagnosis of ARF was totally based on renal biopsy. So overall management alteration in non ATN - ARF patients was $45.94 \%$ (17 out of 37 cases). Thus, early renal biopsy contributes to specific treatment and therefore the outcome of patients with non ATN-ARF.
\end{abstract}

Keywords: Acute Renal failure, Acute tubular necrosis, Amyloidosis, glomerulonephritis, Multiple myeloma, Vasculitis.

\section{Introduction}

The renal biopsy is of fundamental value in discussing the diagnosis and therapy of especially glomerular and vascular causes of acute renal failure. In this study we have included renal biopsies of patients with ARF which on clinical grounds were thought to be due to causes other than acute tubular necrosis (ATN).

Renal biopsy in Acute Renal failure (ARF) is usually indicated in following conditions

1. ARF requiring specific treatment, for example;

i. Glomerulonephritis (GN).

ii. Vasculitis.

iii. Acute interstitial nephritis (AIN).

2. ARF of more subtle onset.

3. When there is no obvious cause for ARF.

4. ARF accompanied by proteinuria or hematuria.

5. When there is evidence of a systemic disease.

\section{Aims and Objectives}

Present study is aimed to determine the contribution of early renal biopsy in diagnosis and management of ARF cases suspected, on clinical grounds to be due to causes other than ATN (non ATNARF).

\section{Materials and Methods}

This prospective and descriptive observational case series study was carried out during the period June 2011 to May 2012. During this period total 336 ARF patients were noted out of which 245 patients were clinically diagnosed as ATN and were excluded from this study. Biopsy was not done in 39 cases. In 15 cases it was a late or postmortem biopsy and were excluded from this study. Mean duration of symptoms to biopsy was $8.08 \pm 4.75$ days. Mean time from admission to biopsy was $4.16 \pm 1.73$ days. Total 37 cases fulfilled the inclusion criteria and are included in this study.

\section{Inclusion Criteria}

Following criterias are applied for inclusion of cases in this study

1. Patients without previously known renal disease.

2. First absolute value of serum creatinine $>2 \mathrm{mg} \%$, OR daily increase in creatinine by $50 \%$ over baseline.

3. Cases where decrease in calculated Glomerular Filtration Rate (GFR) is occurring over days.

4. Cases of unexplained ARF in native kidneys.

5. Cases where renal biopsy was performed within 15 days of onset of symptoms and within 7 days of hospitalization.

\section{Exclusion Criteria}

Following criteria's are applied for exclusion of cases from this study

1. ARF because of suspected acute tubular necrosis (ATN).

2. Cases where renal biopsy was performed later than 7 days after hospitalisation or 15 days later from the onset of symptoms either because of technical difficulties or late referral.

3. Cases of ARF occurring in allograft kidneys were excluded.

\section{Non Invasive Investigative Workup}

Routine Investigations which were carried out on the cases included in this study are: Complete urine analysis, blood urea and serum creatinine estimation, serological test for HIV, HBsAg (Australia antigen) and anti $\mathrm{HCV}$ (hepatitis $\mathrm{C}$ virus). Ultrasonography with Doppler studies were performed in selective cases. 24 hour urine protein excretion was estimated. 
Special Investigations were carried out wherever necessary: Test for Anti-nuclear antibody (ANA), Anti dsDNA antibody, \& anti neutrophil cytoplasmic antibody (ANCA) were performed. Serum compliment (C3 and C4) levels were estimated. Serum protein electrophoresis was done when indicated. In febrile patients blood and urine culture was done. Bone marrow aspiration and trephine biopsy was performed in selected cases. In cases of pulmonary hemorrhages anti glomerular basement membrane (GBM) antibody titres were obtained. Uroflowmetry was carried out where urinary tract obstruction was suspected.
Management was considered altered if:

1. New medication was added to the previous treatment after renal biopsy diagnosis.

2. Previous medication was withdrawn or withheld after renal biopsy diagnosis.

3. Additional investigational procedure was advised and performed after renal biopsy diagnosis.

4. Specific therapeutic procedures were started after kidney biopsy.

Renal biopsy performed with biopsy gun of $16 \mathrm{G}$

(BARD MONOPTY), under USG guidance.

\section{Results and Observation}

Table 1: Showing data of the present study

\begin{tabular}{|l|c|}
\hline \multicolumn{1}{|c|}{ Study period } & June 2011 - May 2012 \\
\hline Total no of ARF patients & 336 \\
\hline Presumed ATN & 245 \\
\hline Biopsy not done & 39 \\
\hline Late or post mortem biopsies & 15 \\
\hline Fulfilled inclusion criteria & 37 \\
\hline Mean duration of symptoms to biopsy & $8.08 \pm 4.75$ days \\
\hline Mean time for admission to biopsy & $4.16 \pm 1.73$ days \\
\hline Clinical and histological diagnosis - matched & 20 \\
\hline Clinical and histological diagnosis - differed & 13 \\
\hline Diagnosis of clinically undiagnosable ARF by biopsy. & 4 \\
\hline
\end{tabular}

Table 2: Depicting 37 cases which satisfied the inclusion criteria and their clinical and biopsy diagnosis

\begin{tabular}{|l|c|c|}
\hline \multicolumn{1}{|c|}{ Diagnosis } & Clinical & Biopsy \\
\hline Proliferative Glomerulonephritis - & - & 11 \\
\hline Lupus nephritis & 2 & 1 \\
\hline Mesangial Hypercellularity & - & 3 \\
\hline IgA Nephropathy & - & 3 \\
\hline Diffuse proliferative & - & 1 \\
\hline Chronic Sclerosing & - & 3 \\
\hline Vasculitis & 2 & 1 \\
\hline Pulmonary renal syndrome & 2 & - \\
\hline Crescentic Glomerulonephritis & - & 6 \\
\hline Anti GBM disease & - & 4 \\
\hline Amyloidosis & 1 & - \\
\hline Membranous & - & 1 \\
\hline Thrombotic Thrombocytopenic Purpura (TTP) & 2 & - \\
\hline Thrombotic Microangiopathy & 2 & 4 \\
\hline HELLP Syndrome & 1 & - \\
\hline Drug induced - & 10 & - \\
\hline ATN & - & 5 \\
\hline AIN & 5 & 2 \\
\hline Clinically undiagnosable & 4 & - \\
\hline Cortical necrosis & - & 1 \\
\hline Myeloma Cast Nephropathy & - & 2 \\
\hline
\end{tabular}

Table 3: Showing 13 cases where renal biopsy diagnosis differed from clinical diagnosis

\begin{tabular}{|l|c|}
\hline \multicolumn{1}{|c|}{ Clinical diagnosis } & Biopsy findings \\
\hline Thrombotic Thrombocytopenic Purpura & Mesangial hypercellularity \& ATN \\
\hline Thrombotic Thrombocytopenic Purpura & Cortical Necrosis \\
\hline
\end{tabular}




\begin{tabular}{|l|c|}
\hline HELLP Syndrome & Thrombotic Microangiopathy \\
\hline Rapidly progressive renal failure & ATN with Acute pyelonephritis \\
\hline Acute Nephritic Syndrome & ATN with IgA Nephropathy \\
\hline Proliferative GN & Chronic Sclerosing GN \\
\hline NSAID induced & Focal proliferative IgA Nephropathy \\
\hline NSAID induced & Class IV Lupus Nephritis \\
\hline Obstructive / NSAID induced & Myeloma Cast Nephropathy \\
\hline AIN/RPRF & Myeloma Cast Nephropathy \\
\hline Drug induced AIN & Crescentic \\
\hline Vasculitis? Polyarteritis nodosa & Thrombotic microangiopathy \\
\hline Vasculitis & Anti GBM disease \\
\hline
\end{tabular}

Table 4: Showing biopsy diagnosis in four cases of clinically undiagnosable ARF:

\begin{tabular}{|l|c|}
\hline Clinical diagnosis & Biopsy findings \\
\hline Clinically undiagnosable ARF & IgA Nephropathy \\
\hline Clinically undiagnosable ARF & IgA Nephropathy \\
\hline Clinically undiagnosable ARF & $\begin{array}{c}\text { End stage renal disease (ESRD) due to Chronic } \\
\text { tubulointerstitial nephritis (CTIN) }\end{array}$ \\
\hline Clinically undiagnosable ARF & Crescentic Glomerulonephritis \\
\hline
\end{tabular}

Table 5: Showing post biopsy treatment alteration

\begin{tabular}{|l|c|}
\hline Steroids started post biopsy & 4 \\
\hline $\begin{array}{l}\text { Bone marrow and chemotherapy } \\
\text { started post biopsy }\end{array}$ & 2 \\
\hline Cytotoxic agents added post biopsy & 5 \\
\hline Plasma exchanges & 3 \\
\hline Steroids stopped & 2 \\
\hline Antibiotics started & 1 \\
\hline Plasma infusions stopped & 1 \\
\hline
\end{tabular}

Table 6: Showing complications of renal biopsies performed in this study

\begin{tabular}{|l|c|}
\hline \multicolumn{1}{|c|}{ Complication } & Number \\
\hline Hematuria post biopsy & 1 \\
\hline Need for transfusion & 0 \\
\hline Clot retention & 1 \\
\hline Surgical intervention & 0 \\
\hline Angiography & 0 \\
\hline
\end{tabular}

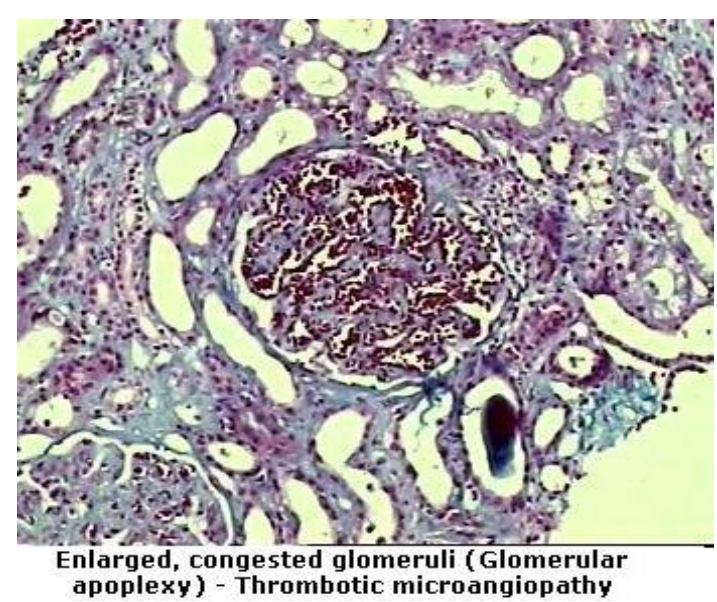

Fig. 1: Masson trichrome, 10X; showing enlarged congested glomeruli (glomerular apoplexy)Thrombotic microangiopathy

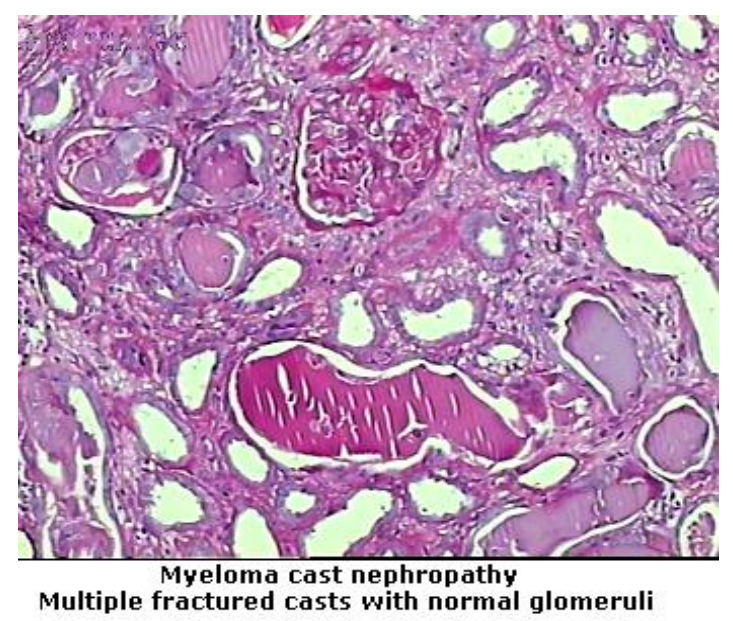

Fig. 2: HE stain, 10X; showing myeloma cast nephropathy. Multiple fractured casts with normal glomeruli

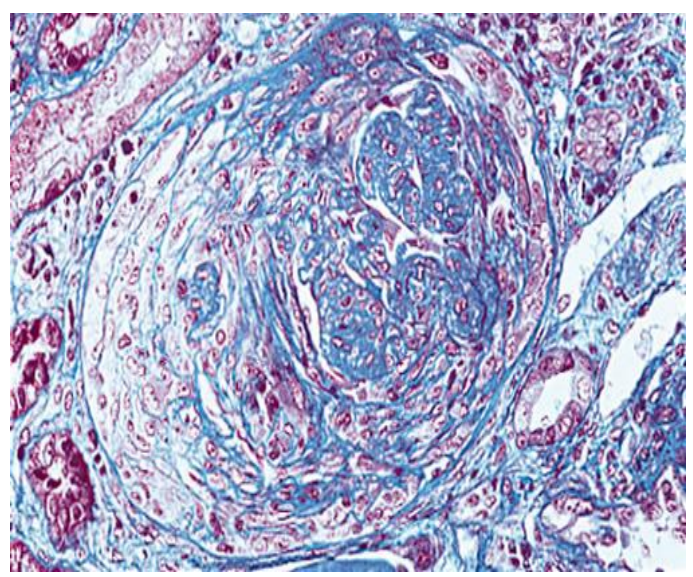

Fig. 3: Masson trichrome, 10X; showing crescentic glomerulonephritis 


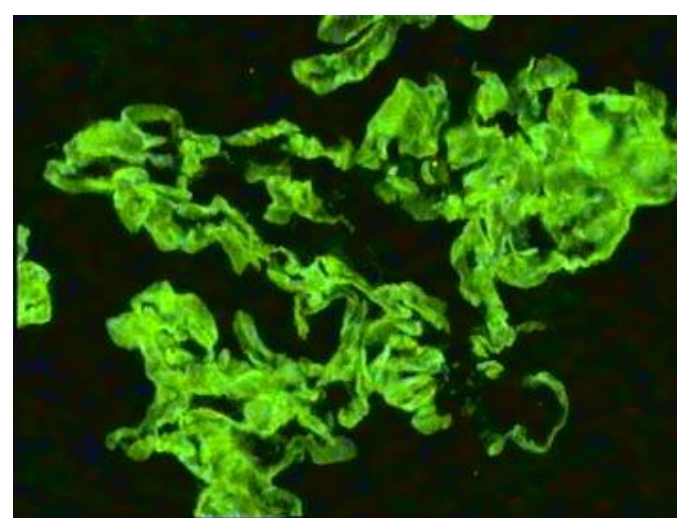

Linear IgG deposits along glomerular capillary wall.

Fig. 4: Immunofluorescence, 10X; Showing linear IgG deposits along glomerular capillary wall

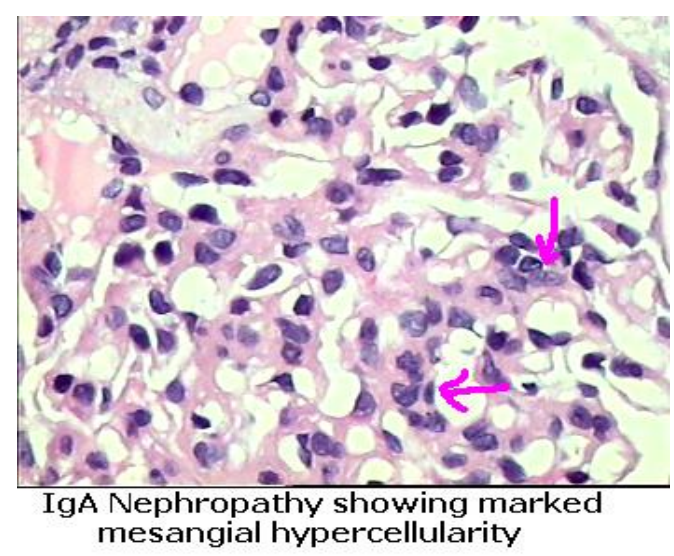

Fig. 5: HE stain, 40X; IgA nephropathy showing marked mesangial hypercellularity

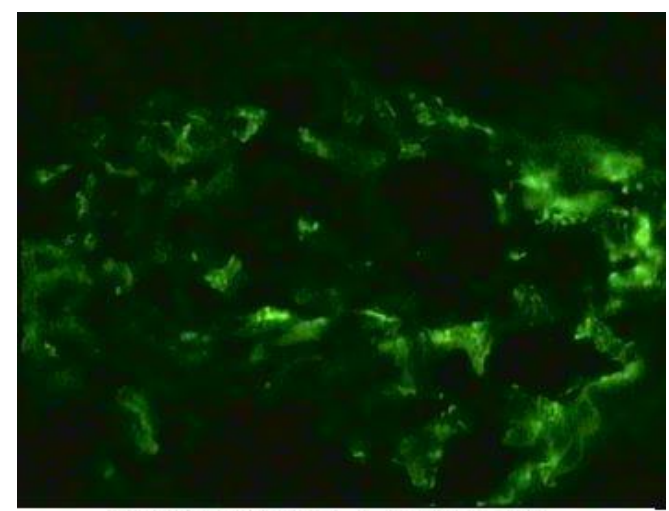

IgA Positive $(+++)$ Diffuse

mesangial deposits are seen.

Fig. 6: Immunofluorescence, 40X; IgA positive (+++) diffuse mesangial deposites are seen

\section{Illustrative Cases}

\section{Case 1:}

1. A 70 year old man came with complaints of pain and swelling in all large joints since 15 days, which was not responding to Non-steroidal AntiInflammatory Drugs (NSAID) and corticosteroids. Patient had generalized myalgia and low grade fever since 7 days and oliguria since 4 days. There was dry gangrene of all toes of Right lower limb since 8 days. There was a petechial rash over both lower limb since 3 days. On examination lower limb and scrotal edema was present. There were rising values of renal function test (RFTs) parameters i.e; urea and creatinine.

2. Taking into consideration above mentioned parameters a working diagnosis of rapidly progressive renal failure (RPRF) most likely secondary to vasculitis was considered. Differential diagnosis of Polyarteritis Nodosa (PAN), ANCA positive vasculitis and cryoglobulinemia was considered.

3. On investigations urine analysis revealed - grade I proteinuria, haematuria (RBC -48 to 50/HPF), granular and RBC casts. 24 hour urinary protein excretion was $990 \mathrm{mg}$. Patient was $\mathrm{HBsAg}$ positive. Test for serum cryoglobulins and ANCA antibodies were negative. Test for anti-nuclear antibody (ANA) was doubtful positive. Anti dsDNA antibody titre was - $4.5 \mathrm{IU} / \mathrm{L}$. Kidney biopsy was performed and diagnosis of thrombotic microangiopathy was confirmed.

\section{Case 2:}

Clinically ARF in a Previously Healthy Person: A 65 year old male with complaints of joint and back pain clinically presented with acute renal failure. On examination patient was dehydrated. Laboratory investigations revealed, Haemoglobin: $5.1 \mathrm{~g} / \mathrm{dl}$, TLC: 7500/mm 3 , DLC: Neutrophil 70\%, Lymphocyte 24\%, eosinophil 04\%, and monocyte 02\%. Peripheral blood smear showed macrocytosis with mild hypochromia. Blood urea: $66 \mathrm{mg} / \mathrm{dl}$, serum creatinine: $3.1 \mathrm{mg} / \mathrm{dl}$, serum total calcium was $6.3 \mathrm{mg} / \mathrm{dl}$, serum total protein: $6.8 \mathrm{~g} / \mathrm{dl}$, serum albumin: $2.8 \mathrm{~g} / \mathrm{dl}$, serum globulin: 4.0 $\mathrm{g} / \mathrm{dl}$. Urine examination showed grade II proteinuria with plenty of pus cells. Toluene sulfonic acid test was negative. Renal biopsy revealed myloma cast nephropathy.

\section{Case 3:}

ARF in a Child: A 10 year old male child presented with sudden decrease in urinary output. History of hemoptysis and hematuria was present. Blood urea was $109 \mathrm{mg} / \mathrm{dl}$ and serum creatinine was $3.2 \mathrm{mg} / \mathrm{dl}$. Anti glomerular basement membrane (GBM) antibody titre was 51.48 (strong positive). Renal biopsy revealed antiGBM disease, consistent with Goodpasture's syndrome.

\section{Case 4:}

A 25 year old male with past history of joint pains in 1994 clinically presented with joint pain and edema since last three months. Urine examination showed grade IV proteinuria, 2 to $3 \mathrm{RBC} / \mathrm{hpf}$ and pus cells 20 to 25/hpf. Renal biopsy revealed lupus nephritis class IV$\mathrm{G}(\mathrm{A})$. 


\section{Case 5:}

A 25 year old male patient presented with haematuria and hypertension. Urine examination revealed grade III proteinuria and plenty of RBC's. There was no history of past illness and no surgical cause of haematuria was found. So renal biopsy was performed. Renal biopsy revealed IgA nephropathy.

\section{Discussion}

This observational, descriptive and prospective study was carried out to assess the effect of knowledge of renal histology on patient management in ARF which were suspected on clinical grounds due to causes other than ATN. 17 out of 37 (45.94\%) were benifited due to renal biopsy diagnosis and the management was altered in these cases. Prednisolone was started in four patients; cytotoxic drugs were added in five patients; prednisolone, cyclophosphamide and plasma exchanges were given in four patients; plasma exchanges alone were carried out in three patients; withholding of initially started medication was possible in two patients and bone marrow biopsy and introduction of chemotherapy was possible in two patients.

Richards et al in $1994^{1}$ carried out similar study in ARF and observed that in 22 out of 31 cases $(70.9 \%)$ the management of the patients altered after renal biopsy diagnosis. The present study reflects the findings of Richards et al $1994^{1}$ and suggest that the knowledge of renal histology is essential in the management of patients with non ATN-ARF.

Jukka Mustonen et al in $1984^{2}$ studied native renal biopsies of 91 patients having acute intrinsic renal failure. They found that the clinical diagnosis of acute tubulo- interstitial renal disease matched with renal biopsy diagnosis in $77 \%$ cases. They also observed that clinical diagnosis of acute glomerulonephritis matched with renal biopsy diagnosis in $56 \%$ cases. According to Jukka Mustonen et al $1984^{2}$ in $15 \%$ cases clinical diagnosis was not possible and renal biopsy was only of diagnostic help in these $15 \%$ cases. In the present study in four out of 37 cases $(10.81 \%)$ clinical diagnosis was not possible and renal biopsy was only diagnostic. Jukka Mestonen et al also observed post biopsy treatment alterations in $20 \%$ cases. In the present study post biopsy treatment alterations was observed in $45.94 \%$ (17 out of 37 ) cases.

\section{Conclusion}

Renal biopsy leads to correct diagnosis in substantial number $(45.94 \%$ cases) of non ATN-ARF patients. Early renal biopsy contributes to specific treatment and therefore affects the outcome of patients with non ATN-ARF. It is a safe, easily performed, diagnostic technique and its complications are minor and very few.

\section{References}

1. Richards NT, Darby S, Howie AJ, Adu D, Michael J. Knowledge of renal histology alters patient management in over $40 \%$ of cases. Nephrol Dial Transplant 1994;9(9):1255-9.

2. Jukka Mustonen, Amos Pasternack, Heikki Helin, Sauli Pystynen, Tapio Tuominen. Renal Biopsy in Acute Renal Failure. Am. J. Nephrol. 4:27-31 (1984).

How to cite this article: Mankar PG, Deshpande S. Early renal biopsy in acute renal failure Expect the unexpected. Ind $\mathrm{J}$ Pathol Oncol, 2018;5(3):370-374. 